\title{
POPULATION DYNAMICS AND IT'S RELEVANT IN MATHEMATICAL MODELLING
}

\section{Marthak Rutu D}

\begin{abstract}
The study of Population dynamics and its relevancy in mathematical modelling to calculate the motion of earth around the sun given the inverse square law of gravitational attraction between them while the geometrical to analyzing dynamical behaviour proved to be a powerful method. Today the study of dynamics reaches far beyond application in celestial mechanics and it has achieved a truly interdisciplinary status.
\end{abstract}

Key Words: Geometrical, celestial, exponential, population dynamics

\section{Introduction :}

The study of dynamical systems has its origins in fifteenth century physics, with Newton's invention of differential equations and solution to the two-body problem; a twobody problem is, for instance, to calculate the motion of earth around the sun given the inverse-square law of gravitational attraction between them (Strogatz, 1994). Newton and others in this time period (such as Euler, Leibniz, Gauss, and Laplace) worked to find analytic solutions to problems of planetary motion; yet, as it turned out, solutions to the three-body problem (e.g., sun, earth, and moon) were nearly impossible to achieve analytically—in contrast to the two-body problem (1994). As a result, other approaches were developed.

In the late 1800s Henri Poincaré developed many of the graphical methods still used today for analyzing the dynamics of systems that extend in complexity beyond the twobody problem. The geometrical approach pioneered by Poincaré proved to be powerful approach to finding a global, qualitative understanding of a system's dynamics (Kaplan \& Glass, 1995; Strogatz, 1994).

While the geometrical approach to analyzing dynamical behaviors proved to be a powerful method, there remained an additional source of analysis that could not be wellharnessed until the 


\section{Rutu D/ Page 71-78}

rise of computing in the 1950s. With the tireless number-crunching capabilities provided by the computer, numerical methods could finally realize a far greater potential. The computer allowed one to develop a more intuitive grasp of nonlinear equations by providing rapid numerical calculations. This advancement in technology, coupled with the geometric methods of analysis, facilitated the surge of developments that occurred in the field of nonlinear dynamics throughout the 1960s and 1970s (Strogatz, 1994).

For example, in 1963, Lorenz discovered the chaotic motion of a strange attractor. He observed that the equations of his three-dimensional system never settled down to an equilibrium state, but rather, they continued to oscillate in an aperiodic fashion (Lorenz, 1963). Additionally, running simulations from different, yet arbitrarily close, initial conditions led to unpredictably different behaviors. Plotted in 3 dimensions, the solutions to his equations fell onto a butterflyshaped set of points (1963). It was later shown that this set contained the properties of a fractal, and his example became a major influence in chaos theory (Strogatz, 1994).

Today, the study of dynamics reaches far beyond applications in celestial mechanics, and it has achieved a truly interdisciplinary status. Significant roles have been established for studying dynamical systems in biology, chemistry, physics, cognitive science, meteorology, the social sciences, finance, philosophy, and so forth. Herein we will be considering dynamical systems solely from the standpoint of population ecology.

The following biographies are of key contributors to the study of population dynamics, with a particular emphasis on individuals whose contributions and influences are most salient in the work outlined in the following chapters, i.e., in continuous ordinary differential equation models of population dynamics. A key work used in outlining this section by Nicolas Bacaër (2011) provides a compact yet thorough account of the historical figures associated with the development of population dynamics. 
Towards Excellence: An Indexed, Refereed \& Peer Reviewed Journal of Higher Education / Marthak

\section{Rutu D/ Page 71-78}

\section{FIBONACCI :}

Leonardo of Pisa, who posthumously became known as Fibonacci, finished writing Liber abaci in 1202, in which he explained various applications of the Arabic number system (decimal) in accounting, unit conversions, interest rates, etc (Sigler, 2002). Appearing as a mere exercise in the midst of unrelated problems, Fibonacci outlined a problem that today would be described as a problem in population dynamics \{Document not in library: (Bacaer, 2011a)\}.

He formulated his question with regard to a pair of mating rabbits and the number of offspring that could be expected after a given period of time. He wrote the following discrete difference equation:

$$
P_{n+1}=P_{n}+P_{n-1}
$$

which states that the number of pairs of rabbits $P_{n+1}$ aftern +1 months is the sum of the number of pairs in month $n$ and of the number of baby pairs in month $n+1$; however, baby rabbits cannot reproduce; therefore, they are considered to be the pairs that were present in month $n-1$.

Fibonacci's rabbit problem was overlooked for several centuries; however, it is now recognized as one of the first models in population dynamics \{Document not in library:

(Bacaer, 2011a)\}. While the rabbit equation (1) turned out to be an unrealistic model (i.e., there are no limitations on growth, no mortality, etc.), the recurrence relation that bears Fibonacci's name has an interesting relationship with naturally occurring geometries, and is found in numerous natural formations ranging from seashells to sunflowers

\{Document not in library: (Bacaer, 2011)\}. The ratio ${ }^{P_{n+1}} / P_{n}$ approaches the so-called golden ratio $\varphi=\frac{1+\sqrt{5}}{2} \approx 1.618$ as $n \rightarrow \infty$. Despite the unrealistic nature of Fibonacci's model with regard to populations, it does share a common property with nearly all population models, namely geometrically increasing growth \{Document not in library: (Bacaer, 2011)\}. 
Towards Excellence: An Indexed, Refereed \& Peer Reviewed Journal of Higher Education / Marthak Rutu D/ Page 71-78

\section{Leonhard Euler:}

Leonhard Euler was a Swiss mathematician born in 1701. He made numerous contributions in the fields of mechanics and mathematics, and is considered to be one of the most prolific mathematicians of his time \{Document not in library: (Bacaer, 2011a)\}. Given the breadth of his work and his display of interest in demography, his work in population dynamics is only natural.

Euler stated that a population $P_{n}$ in year $n$ would satisfy the difference equation

$P_{n+1}=(1+\alpha) P_{n}$

where $n$ is a positive integer and the growth rate $\alpha$ is a positive real number. With an initial condition $P_{0}$, we find the population size in year $n$ by the equation

$P_{n}=(1+\alpha)^{n} P_{0}$

The form of growth assumed by this equation is called geometric growth, (or exponential growth when dealing with continuous equations). As the son of a Protestant minister and having remained in strict religious faith, Euler found this growth model to suit the biblical story in Genesis which held that the entire earth's population descended from very few individuals, namely the three sons of Noah \{Document not in library: (Bacaer, 2011)\}. Despite this ideological alignment, however, Euler recognized that the earth would never sustain such a high rate of growth, given the fact that populations would have climbed upwards to 166 billion individuals in only 400 years. Fifty years after Euler's formulations, Malthus considered the consequences of such growth with regard to human populations in his famous book titled An Essay on the Principle of Population (1798).

\section{DANIEL BERNOULLI:}

Daniel Bernoulli was born in 1700 into a family of already well-established mathematicians: his father Johann Bernoulli and his uncle Jacob Bernoulli. His father did not want him to study mathematics, so Daniel began studying medicine, obtaining his doctorate in 1721 [Document not in library: (Bacaer, 2011a)\}. Within four years, however, he published his first book on mathematics, titled Exercitationes quaedam mathematicae. After his publication, he became involved in a series 
Towards Excellence: An Indexed, Refereed \& Peer Reviewed Journal of Higher Education / Marthak Rutu D/ Page 71-78

of professorships in botany, physiology, and physics, and around the year 1760, Bernoulli undertook studies analyzing the benefits of smallpox inoculation given the associated risk of death from inoculation. His model held the following assumptions:

- The number of susceptible individuals $S(t)$ indicates those uninfected individuals at age $t$ who remain susceptible to the smallpox virus.

- The number of individuals $R(t)$ indicates those whom are infected with the virus but who remain alive at age $t$.

- The total number of individuals $P(t)$ equals the sum of $S(t)$ and $R(t)$.

The model's parameters $q$ and $m(t)$, respectively, represent each individual's probability of becoming infected with smallpox and each individual's probability of dying from other causes. Given these assumptions, Bernoulli derived the following ODEs:

$\frac{d S}{d t}=-q S-m(t) S$

$$
\frac{d R}{d t}=q(1-p) S-m(t) R
$$

The sum of these equations yields

$$
\frac{d P}{d t}=-p q S-m(t) P
$$

and using Eqs. (1) and (2), he yielded the fraction of susceptible individuals at tage by

$\frac{S(t)}{P(t)}=\frac{1}{(1-P) e^{q t}+P}$

Bernoulli estimated the model's parameters using Edmond Halley's life table, which provided the distribution of living individuals for each age \{Document not in library: (Bacaer, 2011)\}. Choosing $q=1 / 8$ per year, and having eliminated $m(t)$ through mathematical trickery, he computed the total number of susceptible people using Eq. (4), finding that approximately $1 / 13$ of 
the population's deaths was expected to be due to smallpox. He further developed his model to examine the costs and benefits of inoculation, which he concluded were undoubtedly beneficialthe life expectancy of an inoculated individual was raised by over three years. Despite these findings, the State never promoted smallpox inoculation, and ironically, the demise of King Louis $X V$ in 1774 was a result of the smallpox virus \{Document not in library: (Bacaer, 2011)\}.

\section{THOMAS ROBERT MALTHUS :}

Thomas Robert Malthus, born 1766, was a British scholar who studied mathematics at Cambridge University, obtaining his diploma in 1791, and six years later becoming a priest of the Anglican Church \{Document not in library: (Bacaer, 2011a)\}.

In 1798 Malthus anonymously published An Essay on the Principle of Population, as It Affects the Future Improvement of Society, With Remarks on the Speculations of Mr.

Godwin, Mr. Condorcet and Other Writers (1798). In his book, he argued that the two named French authors' optimistic views of an ever-progressing society were flawed-particularly, they did not consider the rapid growth of human populations against the backdrop of limited resources (1798). For Malthus, the English Poor Laws, which favored population growth indirectly through subsidized feeding, did not actually help the poor, but to the contrary \{Document not in library: (Bacaer, 2011a)\}. Given the growth of human populations proceeding at a far greater rate than the supply of food, Malthus predicted (albeit, incorrectly) a society plagued by misery and hunger.

The so-called Malthusian growth model is described by the differential equation

$\frac{d N}{d t}=r N$

where the growth of population $N$ is governed by the net intrinsic growth rate parameter $r=b-d$, which is the rate of fertility minus the mortality rate.

Malthus emphasized that this equation holds true in capturing a growing population's dynamics only when growth goes unchecked (Malthus, 1798). However, the continued exponential 
Towards Excellence: An Indexed, Refereed \& Peer Reviewed Journal of Higher Education / Marthak Rutu D/ Page 71-78

growth of human populations against Earth's limited resources, Malthus argued, would ultimately lead to increased human suffering (1798).

Malthus' ideas proved to be influential in the work of numerous individuals, from Verhulst's density-dependent growth model to ideas of natural selection pioneered by Charles Darwin and Alfred Russell Wallace \{Document not in library: (Bacaer, 2011a)\}. 


\section{References}

(1) Mathematical models in population dynemics,by alexander Salisbury

(2) Courchamp, F., Luděk, B., \& Gascoigne, J. (2008). Allee Effects in Ecology and Conservation (p. 256). Oxford: Oxford University Press.

(3) Edelstein-Keshet, L. (2005). Mathematical Models in Biology (p. 586). Philadelphia, PA: SIAM.

(4) Gilpin, M. E., \& Ayala, F. J. (1973). Global models of growth and competition. Proceedings of the National Academy of Sciences of the United States of America, 70(12), 3590-3

(5) Kaplan, D., \& Glass, L. (1995). Understanding Nonlinear Dynamics (p. 440). New York, NY: Springer.

(6) Kot, M. (2001). Elements of Mathematical Ecology (p. 447). Cambridge: Cambridge University Press.

\section{Marthak Rutu D \\ Ph.D Research Scholar \\ Department of Mathematics \\ HNGU, Patan}

